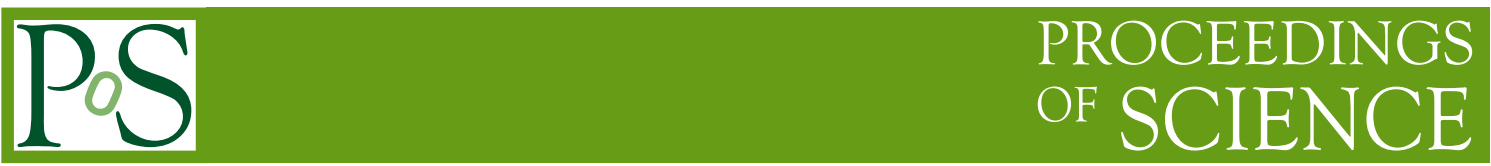

\title{
Positivity Bounds on Vector Boson Scattering
}

\section{Shuang-Yong Zhou*}

Interdisciplinary Center for Theoretical Study, University of Science and Technology of China,

Hefei, Anhui 230026, China

E-mail: zhoushyeustc.edu.cn

\begin{abstract}
While Standard Model Effective Field Theory (SMEFT) systematically categorizes possible new physics beyond the Standard Model, the parameter space of the SMEFT Wilson coefficients is extremely vast in the current standard approach, which hinders experimental searches for new physics. However, there are actually consistent conditions for the Wilson coefficients, arising from requiring that SMEFT or, more precisely, the underlying higher energy theory satisfies some axiomatic principles of quantum field theory. These consistent conditions go by the name of positivity bounds, and the SMEFT parameter space can be significantly reduced by imposing these positivity bounds. Particularly, we show that for the dim- 8 subspace involving vector boson scattering, only about $2 \%$ of the total parameter space satisfies the positivity bounds and thus the majority of the naive SMEFT parameter space is theoretically inconsistent/unphysical.
\end{abstract}

European Physical Society Conference on High Energy Physics - EPS-HEP2019 -

10-17 July, 2019

Ghent, Belgium

${ }^{*}$ Speaker. 


\section{Introduction and Summary}

New physics beyond the Standard Model can be systematically parametrized within the framework of Standard Model Effective Field Theory (SMEFT), which is the renormalizable Standard Model Lagrangian augmented by higher dimensional operators consistent with the symmetries of the Standard Model. In the standard approach, in the absence of any detection of new phenomena, the various coefficients in front of the higher dimensional operators, often called Wilson coefficients, are a priori allowed to take arbitrary values, encoding various different kinds of new physics beyond the Standard Model. As a result, the parameter space of SMEFT is extremely large.

However, not every values are allowed for the Wilson coefficients, if SMEFT is assumed to have a theoretically consistent ultra-violet (UV) completion. Specifically, there are so-called positivity bounds $[1,2]$ that the Wilson coefficients have to satisfy. As an EFT, SMEFT breaks down (i.e., violates perturbative unitarity) at the cutoff scale $\Lambda$, beyond which SMEFT loses its validity and a UV completion is needed. The UV complete theory should satisfy the usual axiomatic principles of quantum field theory such as unitarity and analyticity. Using these properties, one can express the scattering amplitude of the UV theory in terms of an integral of the imaginary part of the amplitude along the real axis of $s$ ( $s$ being the Mandelstam variable), which is called the dispersion relation (cf. the left of Fig. 1 and Section 2), and eventually derive a set of inequalities that involve the amplitude and its derivatives w.r.t. the Mandelstam variables. Thus, from these purely theoretical considerations, one can extract (or bootstrap) a number of physical constraints on the Wilson coefficients. We would like to emphasize that the positivity bounds are different from the unitarity bounds, and in particular analyticity plays an important role in deriving these positivity bounds. In summary, not every set of Wilson coefficients can define a consistent EFT and the set of physical Wilson coefficients must satisfy the positivity bounds.

The positivity bounds have dramatic impacts on reducing the parameter space of SMEFT. In [3, 4], we apply the positivity bounds in leading order vector boson scattering (VBS, i.e., scattering of $W^{+}, W^{-}, Z$ and $\gamma$ ) to constrain anomalous quartic gauge couplings (aQGCs), and find that the positivity bounds exclude the majority of the parameter space of the 18 aQGC related dim- 8 operators (cf. Section 3), and only about $2 \%$ of the total parameter space satisfies the positivity bounds. The positivity constraints on the dim-8 Wilson coefficients are displayed in Section 4, ready to be used to check whether a given set of Wilson coefficients satisfies the positivity bounds.

The reason that we neglect the aQGC related dim- 6 operators is twofold. (1) At leading order, all the dim- 6 operators contribute negatively to the VBS positivity bounds [3]. That is, all the VBS positivity inequalities can be schematically written in the form of $A_{8}-A_{6}>0$, where $A_{8} \sim \mathscr{O}\left(\Lambda^{-4}\right)$ stands for dim- 8 contributions and $A_{6} \sim \mathscr{O}\left(\Lambda^{-4}\right)$ for contributions with 2 dim- 6 three-point vertices; by explicit calculations, one can show that $A_{6}>0$ for all the nontrivial bounds, so $A_{8}>0$ is weaker than $A_{8}-A_{6}>0$ but still a valid bound. This also implies that SMEFT would be inconsistent with only dim- 6 operators but no dim- 8 operators. (2) While QGCscan be affected by dim- 6 operators, it can be expected that the dim- 6 effects will be first detected in other processes such as in Higgs production and decay, diboson production or vector boson fusion. Nevertheless, we have given the full positivity bounds with all the dim- 6 contributions included in $[3,4]$.

The foundation of the positivity bounds relies on the aforementioned axiomatic principles of quantum field theory. Those principles have been accurately tested by various experiments at the 

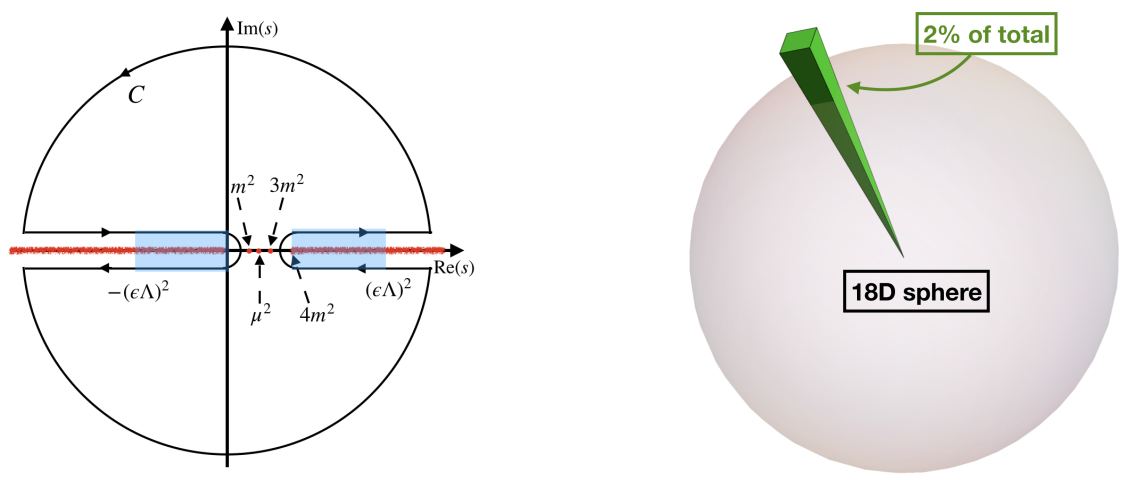

Figure 1: (Left) Dispersion relation contour in the complex $s$ plane. The red lines are branch cuts. The light blue parts can be subtracted since they can be computed within the low energy EFT. (Right) The physical subspace (only about 2\%) of the SMEFT parameter space for the aQGC related dim- 8 operators. This is only a cartoon illustration: the sphere represents a $18 \mathrm{D}$ sphere and the green solid angle is the allowed region.

energies currently accessible, but are of course assumptions at higher energies. So reversing the argument, another usage of these positivity bounds is to test the axiomatic principles of quantum field theory by looking for possible violations of these bounds.

\section{Positivity Bounds}

In this section, we shall outline the derivation of the improved forward limit positivity bound for the toy case of scattering of scalar particles with mass $m$, which captures the essential idea but without further technical complications. For more details and the adaption of the proof in the context of SMEFT, the reader may refer to Section 2 of [4].

The positivity bounds can be derived by assuming the underlying UV complete theory of the EFT satisfies the most cherished principles of quantum field theory, most notably Lorentz invariance, unitarity, locality, analyticity and crossing symmetry. We shall focus on 2-to-2 elastic scattering. By Lorentz invariance, a scalar amplitude must be a Lorentz invariant function of the Mandelstam variables $s$ and $t\left(u=4 m^{2}-s-t\right)$. A textbook application of unitarity is the optical theorem, which states that the imaginary part of the amplitude in the forward limit is related to the total cross section via $\operatorname{Im} A(s, 0)=\sqrt{s\left(s-4 m^{2}\right)} \sigma_{\text {total }}(s)$. This implies that in the physical region $s>4 m^{2}$ we have $\operatorname{Im} A(s, 0)>0$. A bit more sophisticated application of unitary (as well as locality) implies the existence of the Froissart bound [5]: $|A(s, 0)|<c s \ln ^{2} s$, as $s \rightarrow \infty$, where $c$ is a constant. Now, analyticity tells us that when extended to the complex domain, $A(s, 0)$ is analytic except on on the real $s$ axis, which contains possible simple poles at $m^{2}$ and $3 m^{2}$ and branch cuts above $4 m^{2}$ and below 0 . Therefore, by Cauchy's integral formula, we can define a quantity

$$
f\left(\mu^{2}\right) \equiv \frac{1}{2 \pi i} \oint_{C} \mathrm{~d} s \frac{A(s, 0)}{\left(s-\mu^{2}\right)^{3}},
$$

where the contour $C$ runs around the branch cuts and the infinity (cf. the left of Fig. 1). The Froissart bound implies that the contributions from the infinite semi-circles vanish, and by complex analysis 
(or the Schwarz reflection principle) we have $\operatorname{Disc} A(s, 0)=2 i \operatorname{Im} A(s, 0)$, so we have

$$
f\left(\mu^{2}\right)=\frac{1}{2 \pi i}\left(\int_{-\infty}^{0}+\int_{4 m^{2}}^{+\infty}\right) \mathrm{d} s \frac{\operatorname{Disc} A(s, 0)}{\left(s-\mu^{2}\right)^{3}}=\frac{1}{\pi} \int_{4 m^{2}}^{\infty} \mathrm{d} s\left[\frac{\operatorname{Im} A(s, 0)}{\left(s-\mu^{2}\right)^{3}}+\frac{\operatorname{Im} A^{(u)}(s, 0)}{\left(s+\mu^{2}-4 m^{2}\right)^{3}}\right],
$$

where $\operatorname{Im} A^{(u)}(s, 0)$ is the $u$-channel amplitude. The optical theorem tells us that $\operatorname{Im} A(s, 0)>0$ for $s>4 m^{2}$, and by crossing symmetry, we know also that $\operatorname{Im} A^{(u)}(s, 0)>0$ for $s>4 m^{2}$, so we have proven that

$$
f\left(\mu^{2}\right)>0 \text { for } 0<\mu^{2}<4 m^{2} .
$$

Now, crucially, by Cauchy's theorem, $f\left(\mu^{2}\right)$ equals to the sum of the residues of the simple poles within contour $C, f\left(\mu^{2}\right)=\sum_{C} \operatorname{Res}\left[A(s, 0) /\left(s-\mu^{2}\right)^{3}\right]$, and since the poles can be determined at low energies, $f\left(\mu^{2}\right)$ can be computed to a desired accuracy within the EFT and thus $f\left(\mu^{2}\right)>0$ gives rise to constraints on the Wilson coefficients.

For the second equality of Eq. (2.2), the integral on the right hand side is from $4 \mathrm{~m}^{2}$ to infinity. But since we can compute $\operatorname{Im} A(s, 0)$ within the EFT, an improvement on the bound $(2.3)$ is to subtract the integration from $4 m^{2}$ to $(\varepsilon \Lambda)^{2}$,

$$
f_{\varepsilon \Lambda}\left(\mu^{2}\right)=f\left(\mu^{2}\right)-\frac{1}{\pi} \int_{4 m^{2}}^{(\varepsilon \Lambda)^{2}} \mathrm{~d} s\left[\frac{\operatorname{Im} A(s, 0)}{\left(s-\mu^{2}\right)^{3}}+\frac{\operatorname{Im} A^{(u)}(s, 0)}{\left(s+\mu^{2}-4 m^{2}\right)^{3}}\right],
$$

where $\varepsilon \lesssim 1$ (see the light blue regions in the left of Fig. 1). Since the integrand is positive definite from $4 m^{2}$ all the way up to $+\infty$, the quantity $f_{\varepsilon \Lambda}\left(\mu^{2}\right)$ is still positive. This improvement on the bounds turns out to be useful for constraining aQGC Wilson coefficients in SMEFT with a high cutoff scale [4].

\section{Dim-8 operators in VBS}

The SMEFT Lagrangian is given by

$$
\mathscr{L}_{\text {SMEFT }}=\mathscr{L}_{\text {SM }}+\sum_{i} \frac{c_{i} \mathscr{O}_{i}^{(6)}}{\Lambda^{2}}+\sum_{i} \frac{f_{i} \mathscr{O}_{i}^{(8)}}{\Lambda^{4}}+\cdots,
$$

where $\Lambda$ is the cutoff and $\mathscr{L}_{\mathrm{SM}}$ is the Standard Model Lagrangian. SMEFT shares the same particle content, gauge group structure and global symmetries as the Standard Model. We focus on vector boson scattering, and the relevant dim-8 operators are [6]

$$
\begin{array}{rlrl}
\mathscr{O}_{S, 0} & =\left[\left(D_{\mu} \Phi\right)^{\dagger} D_{v} \Phi\right]\left[\left(D^{\mu} \Phi\right)^{\dagger} D^{v} \Phi\right] & & \\
\mathscr{O}_{S, 1}=\left[\left(D_{\mu} \Phi\right)^{\dagger} D^{\mu} \Phi\right]\left[\left(D_{v} \Phi\right)^{\dagger} D^{v} \Phi\right] & & \mathscr{O}_{T, 0}=\operatorname{Tr}\left[\hat{W}_{\mu \nu} \hat{W}^{\mu v}\right] \operatorname{Tr}\left[\hat{W}_{\alpha \beta} \hat{W}^{\alpha \beta}\right] \\
\mathscr{O}_{S, 2}=\left[\left(D_{\mu} \Phi\right)^{\dagger} D_{v} \Phi\right]\left[\left(D^{v} \Phi\right)^{\dagger} D^{\mu} \Phi\right] & & \mathscr{O}_{T, 1}=\operatorname{Tr}\left[\hat{W}_{\alpha v} \hat{W}^{\mu \beta}\right] \operatorname{Tr}\left[\hat{W}_{\mu \beta} \hat{W}^{\alpha v}\right] \\
\mathscr{O}_{M, 0}=\operatorname{Tr}\left[\hat{W}_{\mu v} \hat{W}^{\mu v}\right]\left[\left(D_{\beta} \Phi\right)^{\dagger} D^{\beta} \Phi\right] & \mathscr{O}_{T, 2}=\operatorname{Tr}\left[\hat{W}_{\alpha \mu} \hat{W}^{\mu \beta}\right] \operatorname{Tr}\left[\hat{W}_{\beta v} \hat{W}^{v \alpha}\right] \\
\mathscr{O}_{M, 1}=\operatorname{Tr}\left[\hat{W}_{\mu v} \hat{W}^{v \beta}\right]\left[\left(D_{\beta} \Phi\right)^{\dagger} D^{\mu} \Phi\right] & \mathscr{O}_{T, 5}=\operatorname{Tr}\left[\hat{W}_{\mu v} \hat{W}^{\mu v}\right] \hat{B}_{\alpha \beta} \hat{B}^{\alpha \beta} \\
\mathscr{O}_{M, 2}=\left[\hat{B}_{\mu v} \hat{B}^{\mu v}\right]\left[\left(D_{\beta} \Phi\right)^{\dagger} D^{\beta} \Phi\right] & \mathscr{O}_{T, 6}=\operatorname{Tr}\left[\hat{W}_{\alpha v} \hat{W}^{\mu \beta}\right] \hat{B}_{\mu \beta} \hat{B}^{\alpha v} \\
\mathscr{O}_{M, 3}=\left[\hat{B}_{\mu v} \hat{B}^{v \beta}\right]\left[\left(D_{\beta} \Phi\right)^{\dagger} D^{\mu} \Phi\right] & \mathscr{O}_{T, 7}=\operatorname{Tr}\left[\hat{W}_{\alpha \mu} \hat{W}^{\mu \beta}\right] \hat{B}_{\beta v} \hat{B}^{v \alpha} \\
\mathscr{O}_{M, 4}=\left[\left(D_{\mu} \Phi\right)^{\dagger} \hat{W}_{\beta v} D^{\mu} \Phi\right] \hat{B}^{\beta v} & \mathscr{O}_{T, 8}=\hat{B}_{\mu v} \hat{B}^{\mu v} \hat{B}_{\alpha \beta} \hat{B}^{\alpha \beta} \\
\mathscr{O}_{M, 5}=\frac{1}{2}\left[\left(D_{\mu} \Phi\right)^{\dagger} \hat{W}_{\beta v} D^{v} \Phi\right] \hat{B}^{\beta \mu}+h c & \mathscr{O}_{T, 9}=\hat{B}_{\alpha \mu} \hat{B}^{\mu \beta} \hat{B}_{\beta v} \hat{B}^{v \alpha}, \\
\mathscr{O}_{M, 7}=\left[\left(D_{\mu} \Phi\right)^{\dagger} \hat{W}_{\beta v} \hat{W}^{\beta \mu} D^{v} \Phi\right] & &
\end{array}
$$

where $\hat{W}^{\mu v} \equiv i g \frac{\sigma^{I}}{2} W^{I, \mu v}, \hat{B}^{\mu v} \equiv i g^{\prime} \frac{1}{2} B^{\mu v}$ and the Higgs field should be expanded around the broken vacuum $\Phi=\left(0, \frac{v+h}{\sqrt{2}}\right)^{T}$. 


\section{Results}

The VBS positivity bounds were first given with polarization vectors in [3]. These early bounds require the user to numerically evaluate the bounds for all possible polarizations to pick out the strongest ones. In [4], we have analytically eliminated the polarizations in the positivity bounds, and the resulting bounds are much easier to use. These final bounds are of two kinds:

(1) the linear bounds

$$
\sum_{j}\left(M_{S}\right)_{i j} F_{S, j}>0, \quad \sum_{j}\left(M_{M}\right)_{i j} F_{M, j}>0, \quad \sum_{j}\left(M_{T}\right)_{i j} F_{T, j}>0,
$$

(2) the nonlinear bounds

$$
\begin{gathered}
32\left(2 F_{S, 0}+F_{S, 1}+F_{S, 2}\right)\left(2 F_{T, 0}+F_{T, 1}+F_{T, 2}\right) \\
-\max \left(0,4 F_{M, 0}+F_{M, 1}-4 F_{M, 0}+3 F_{M, 1}-2 F_{M, 7}\right)^{2}>0, \\
\quad 8\left(2 F_{S, 0}+F_{S, 1}+F_{S, 2}\right)\left(8 F_{T, 0}+12 F_{T, 1}+5 F_{T, 2}\right) \\
-\max \left(0,4 F_{M, 0}+F_{M, 1},-4 F_{M, 0}+3 F_{M, 1}-2 F_{M, 7}\right)^{2}>0, \\
8\left(F_{S, 0}+F_{S, 1}+F_{S, 2}\right)\left[4 c_{W}^{8}\left(2 F_{T, 0}+2 F_{T, 1}+F_{T, 2}\right)+2 c_{W}^{4} s_{W}^{4}\left(2 F_{T, 5}+2 F_{T, 6}+F_{T, 7}\right)+s_{W}^{8}\left(2 F_{T, 8}+F_{T, 9}\right)\right] \\
-\max \left(0,2\left(2 c_{W}^{4} F_{M, 0}+F_{M, 2} s_{W}^{4}-F_{M, 4} s_{W}^{4}+F_{M, 4} s_{W}^{2}\right),-c_{W}^{4}\left(4 F_{M, 0}-2 F_{M, 1}+F_{M, 7}\right)-2 c_{W}^{2} F_{M, 4} s_{W}^{2}\right. \\
\left.-s_{W}^{4}\left(2 F_{M, 2}-F_{M, 3}\right)-F_{M, 5}\left(s_{W}^{2}-s_{W}^{4}\right)\right)^{2}>0, \\
16\left(F_{S, 0}+F_{S, 2}\right)\left[4 c_{W}^{4}\left(4 F_{T, 1}+F_{T, 2}\right)+s_{W}^{4}\left(4 F_{T, 6}+F_{T, 7}\right)\right]-\max \left(0,2 c_{W}^{2} F_{M, 7}+F_{M, 5} s_{W}^{2}+4 F_{M, 4} s_{W}^{2}\right. \\
-2 \sqrt{\left(2 F_{M, 1}-F_{M, 7}\right)\left(c_{W}^{4}\left(2 F_{M, 1}-F_{M, 7}\right)+c_{W}^{2} F_{M, 5} s_{W}^{2}+F_{M, 3} s_{W}^{4}\right)+4 F_{M, 4} s_{W}^{2}},-2 c_{W}^{2} F_{M, 7} \\
\left.-F_{M, 5} s_{W}^{2}-4 F_{M, 4} s_{W}^{2}-2 \sqrt{\left(2 F_{M, 1}-F_{M, 7}\right)\left(c_{W}^{4}\left(2 F_{M, 1}-F_{M, 7}\right)+c_{W}^{2} F_{M, 5} s_{W}^{2}+F_{M, 3} s_{W}^{4}\right)}\right)^{2}>0,
\end{gathered}
$$

where $s_{W} \equiv \sin \theta_{W}, c_{W} \equiv \cos \theta_{W}, \theta_{W}$ being the weak angle, $F_{S, i} \equiv f_{S, i}, F_{M, i} \equiv e^{2} f_{M, i}, F_{T, i} \equiv e^{4} f_{T, i}$ are rescaled Wilson coefficients and

$$
\begin{aligned}
M_{S} & =\left(\begin{array}{lll}
2 & 1 & 1 \\
1 & 1 & 1 \\
1 & 0 & 1
\end{array}\right), \quad M_{M}=\left(\begin{array}{cccccccc}
0 & -2 c_{W}^{4} & 0 & -s_{W}^{4} & 0 & s_{W}^{2} c_{W}^{2} & c_{W}^{4} \\
0 & -2 c_{W}^{4} & 0 & -s_{W}^{4} & 0 & -s_{W}^{2} c_{W}^{2} & c_{W}^{4} \\
0 & -2 & 0 & 0 & 0 & 0 & 1 \\
0 & -2 & 0 & -1 & 0 & 1 & 1 \\
0 & -2 & 0 & -1 & 0 & -1 & 1
\end{array}\right), \\
M_{T} & =\left(\begin{array}{ccccccccc}
0 & 0 & 1 & 0 & 0 & 0 & 0 & 0 \\
0 & 2 & 1 & 0 & 0 & 0 & 0 & 0 \\
2 & 1 & 1 & 0 & 0 & 0 & 0 & 0 \\
8 & 12 & 5 & 0 & 0 & 0 & 0 & 0 \\
8 c_{W}^{8} & 8 c_{W}^{8} & 4 c_{W}^{8} & 4 c_{W}^{4} s_{W}^{4} & 4 c_{W}^{4} s_{W}^{4} & 2 c_{W}^{4} s_{W}^{4} & 2 s_{W}^{8} & s_{W}^{8} \\
0 & 0 & 4 c_{W}^{8} & 0 & 0 & 2 c_{W}^{4} s_{W}^{4} & 0 & s_{W}^{8} \\
0 & 0 & 4 c_{W}^{4} & 0 & 0 & s_{W}^{4} & 0 & 0 \\
0 & 16 c_{W}^{4} & 4 c_{W}^{4} & 0 & 4 s_{W}^{4} & s_{W}^{4} & 0 & 0 \\
0 & 0 & 4 & 0 & 0 & 1 & 0 & 0 \\
0 & 16 & 4 & 0 & 4 & 1 & 0 & 0 \\
0 & 0 & 8 c_{W}^{4} & 0 & 0 & \left(c_{W}^{2}-s_{W}^{2}\right)^{2} & 0 & 2 s_{W}^{4} \\
32 c_{W}^{4} & 32 c_{W}^{4} & 16 c_{W}^{4} & -16 c_{W}^{2} s_{W}^{2} & 4\left(c_{W}^{2}-s_{W}^{2}\right)^{2} & 1-8 s_{W}^{2} c_{W}^{2} & 8 s_{W}^{4} & 4 s_{W}^{4} \\
0 & 0 & 4 & 0 & 0 & 2 & 0 & 1 \\
8 & 8 & 4 & 4 & 4 & 2 & 2 & 1
\end{array}\right)
\end{aligned}
$$




\section{Demostrations and comparisons with experiments}

We have mentioned that these positivity bounds significantly reduce the physically available parameter space of aQGCs. Since both the linear and nonlinear bounds are homogeneous algebraically in the Wilson coefficients, the bounds carve out a small, connected solid angle (about $2 \%$ ) in a 18 dimensional sphere. Moreover, the solid angle is convex, since the physical parameter region is the convex hull of all the possible constraints.
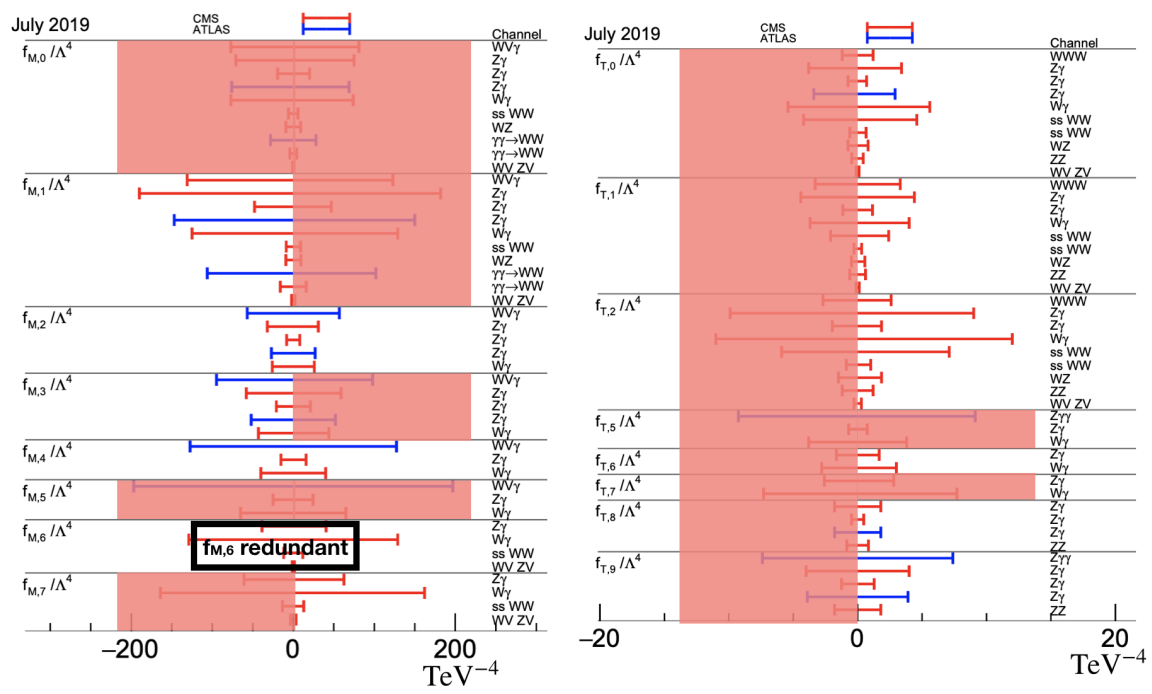

Figure 2: 1D positivity bounds. Only one of the Wilson coefficients are kept nonzero, despite being theoretically unmotivated. The error bars for aQGC Wilson coefficients are from CMS (red) and ATLAS (blue) at $95 \%$ C.L., as summarized in https://twiki.cern.ch/twiki/bin/view/CMSPublic/PhysicsResultsSM PaTGC\#aQGC_Results. The red regions are excluded by the positivity bounds.

We can not draw a 18 dimensional parameter space, but we may check how the positivity bounds look like in lower dimensional slices. As global fits are inherently difficult from the experimental point of view, 1D or 2D constraints (i.e., all the Wilson coefficients are set to zero except for 1 or 2 of them being nonzero) are the standard output from experiments. So it is in these lowest dimensional cases that we can compare to the experimental results; see Fig. 2 and Fig. 3. For more details, see [4]. We would like to emphasize that in a consistent EFT approach where all the Wilson coefficients are allowed to nonzero, some of the lower dimensional bounds may be relaxed. However, the generic feature is unchanged that the positivity bounds significantly reduce the physical parameter space of SMEFT, as can be seen in the right of Fig. 1.

\section{References}

[1] A. Adams, N. Arkani-Hamed, S. Dubovsky, A. Nicolis and R. Rattazzi, Causality, analyticity and an IR obstruction to UV completion, JHEP 10 (2006) 014, [hep-th / 0602178 ].

[2] C. de Rham, S. Melville, A. J. Tolley and S.-Y. Zhou, UV complete me: Positivity Bounds for Particles with Spin, JHEP 03 (2018) 011, [1706.02712]. 

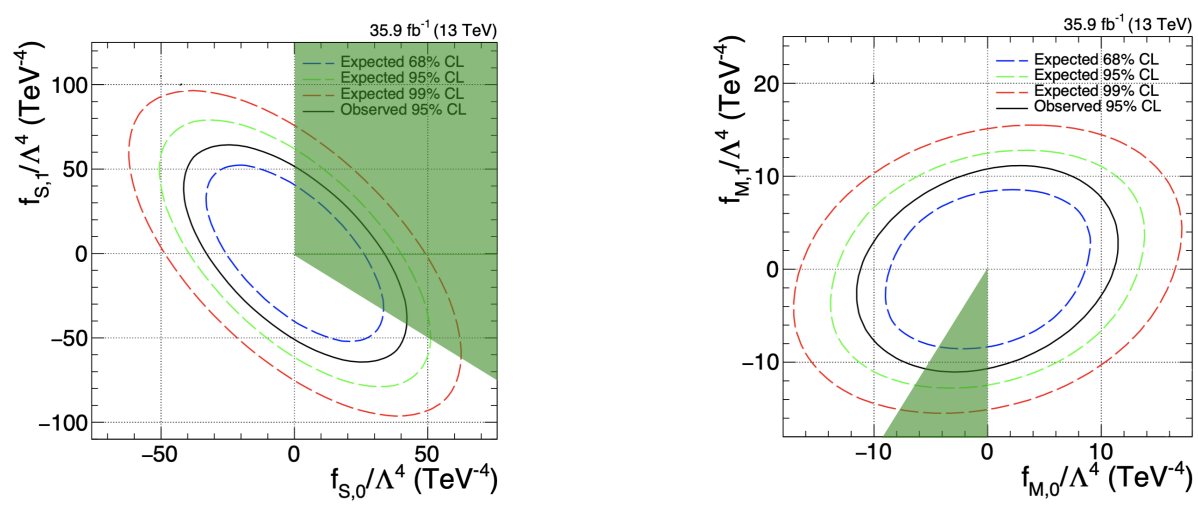

Figure 3: $2 \mathrm{D}$ positivity bounds. The contours are from the CMS $W Z j j$ analysis [7], whilst only the green shaded areas satisfy the positivity bounds.

[3] C. Zhang and S.-Y. Zhou, Positivity bounds on vector boson scattering at the LHC, Phys. Rev. D 100 (2019) 095003, [1808.00010].

[4] Q. Bi, C. Zhang and S.-Y. Zhou, Positivity constraints on aQGC: carving out the physical parameter space, JHEP 06 (2019) 137, [1902 . 08977 ].

[5] M. Froissart, Asymptotic behavior and subtractions in the Mandelstam representation, Phys. Rev. 123 (1961) 1053-1057.

[6] C. Degrande et al, Monte Carlo tools for studies of non-standard electroweak gauge boson interactions in multi-boson processes: A Snowmass White Paper, in Proceedings of 2013 Community Summer Study on the Future of U.S. Particle Physics (CSS2013) (2013). 1309.7890.

[7] CMS collaboration, Measurement of electroweak WZ production and search for new physics in pp collisions at $\operatorname{sqrt}(s)=13 \mathrm{TeV}$, Tech. Rep. CMS-PAS-SMP-18-001, CERN, Geneva, 2018. 